\title{
The potential of eHealth Apps to Support Targeted Complex Health Messages
}

\author{
Kayla J. Heffernan ${ }^{1}$, Shanton Chang ${ }^{1 *}$, Skye T. Maclean ${ }^{1}$, Emma T. Callegari ${ }^{1}$, Suzanne M. Garland ${ }^{2}$, Nicola Reavley ${ }^{1}$, George Varigos ${ }^{1}$ and John D. Wark $^{1}$ \\ ${ }^{1}$ University of Melbourne, Australia \\ ${ }^{2}$ Royal Women's Hospital, Australia \\ *Corresponding author: Shanton Chang, Assistant Dean, Melbourne School of Engineering, University of Melbourne, Australia, Tel: 61383441583; E-mail: \\ shanton.chang@unimelb.edu.au
}

Received date: September 8, 2014; Accepted date: October 6, 2014; Published date: October 8, 2014

Copyright: (c) 2014 Heffernan KJ et al. This is an open-access article distributed under the terms of the Creative Commons Attribution License, which permits unrestricted use, distribution, and reproduction in any medium, provided the original author and source are credited.

\begin{abstract}
With the advent of ubiquitous smartphone devices, health apps have become common, allowing the delivery of services and health messages to target populations, right in their pockets. Therefore, the use of these devices has potential to move information dissemination beyond just face-to-face consultations. This research highlights the lessons learned from the Safe-D (Safe vitamin D) case study to develop an Apple and Android app to safely improve vitamin D status in young women. The Safe-D App aims to assist individuals in a more personal manner, through self-monitoring and personalized relevant health messages that are dependent on the individual's monitored behavior. This paper will provide guidelines for message construction, along with recommendations to deliver targeted messages.
\end{abstract}

Keywords: Health apps; Message delivery

\section{Introduction}

Information dissemination and recommendations by clinicians are limited by short consultations and shortcomings of traditional media [1]; conversely, smartphones enable portable, large-scale, information access $[2,3]$. Smartphones are carried on a person most of the day, allowing continual access to recommendations and information. The ubiquity of smartphones has given rise to health apps, programs that are downloaded to a mobile device; there were 97,000 health apps in major app stores in 2013 [4]. These apps have the "potential to transform the way the public manage (sic) their health" [5]; health information delivery in public health has the potential to be more targeted with these new technologies. Dr. Brian Levine and Dr. Dan Goldschlag propose that these may be advantageous and the way of the future: "it is not unreasonable to envision a day when we not only write a prescription for a medication, but also prescribe an application" [6]. In fact, in the United Kingdom, the National Health Service has created a Health Apps Library, with a clear review process to advise and ensure a collection of Health apps that are clinically sound (www.apps.nhs.uk). However, not all these health apps are interactive, or even when interactive, they tend to provide "one size fits all" delivery of information. Encouragingly, a smaller but growing collection of health apps is starting to provide more personalized information to the users.

This paper seeks to outline the process for determining the creation and delivery of personalized health messages to target users. This research leveraged the Young Female Health Initiative (YFHI) Safe-D case study to develop an Apple and Android app, also named "Improving vitamin D status and related health in young women" (Safe-D), to safely improve vitamin D status in young women aged 16-25 years. This is an example of a health app designed to assist individuals through self-monitoring and personalized relevant health messages that are dependent on the individual's monitored behavior. The interactive Safe-D app is compared with the traditional information delivery in the SunSmart app. Thus, this paper will present the process and findings of developing an app for personalized message delivery, an important area for health service providers to explore.

\section{Background}

Paper-based, SMS and other traditional interventions are, in many ways, deficient in comparison to health apps. Traditional methods are static, thus cannot provide two-way information. The ubiquity of smartphones and proliferation of health apps suggests that they have the potential to help improve health [7]. Smartphones have many benefits over human persuaders including: greater persistence than otherwise acceptable; less invasive prompting [8]; access that human persuaders cannot gain; and inclusion of rich media such as audio, images and video $[6,8]$. Traditional interventions often rely on onesize-fits-all prompts [9]; Health apps afford the two-way information exchange required for interactive, tailored recommendations that are most effective at motivation and education [10-13], providing customized and personalized service delivery. Additionally, health apps enable large-scale interventions [1] at no, or little, additional cost [8]; such would be difficult, if not impossible, by traditional means. The Safe-D study will explore whether the Safe-D health app can improve vitamin D status in young women as well as, or better than, supplementation and/or brochure-based advice on achieving adequate sun exposure. Delivering complex messages simply is essential so that they are understood by the target audience, however it is also important that the messages themselves are personalized. Borsboom [9] indicates tailored solutions are "significantly more effective at improving healthcare outcomes" [9] and tailored information can lead to safer, less cumbersome, interventions. The literature provides support that tailored messages are most effective at [7,10-11].

\section{The Safe-D case study}

While improving health is rarely easy, some health problems require complex messaging, making interventions additionally 
difficult. For example, smoking cessation interventions must decrease smoking over time. The goal is difficult in practice but the message is simple: smoke less and stop. Conversely, achieving adequate vitamin D synthesis, like many health problems, requires complex messaging and is subject to a number of confounding factors. Few foods contain appreciable amounts of vitamin D, only oily fish, sun exposed mushrooms and eggs [12]. In Australia, margarine is fortified with vitamin D [13]; however, this alone is not enough to achieve sufficient levels. Green et al. [13] acknowledge that changing eating patterns to meet vitamin D requirements is not practical and that barriers exist to supplementation [13]. Therefore, sun exposure is essential, as ultra violet (UV) radiation is the primary source of vitamin D [12]. UV, however, varies based on latitude, season and time, cloud cover and pollution. In addition to these environmental factors, vitamin D synthesis is affected by skin pigmentation; darker pigmented skin requires longer exposure than lighter skin to synthesize the same amount of vitamin D [14]. Vitamin D from sunlight is dependent on exposed skin; the greater area covered, the less there is available for synthesis. Use of sunscreen has similar impact, diminishing UV absorption and thus vitamin D synthesis [14]. The factors that affect individualized sun exposure recommendations are depicted in Figure 1 Factors affecting exposure time.

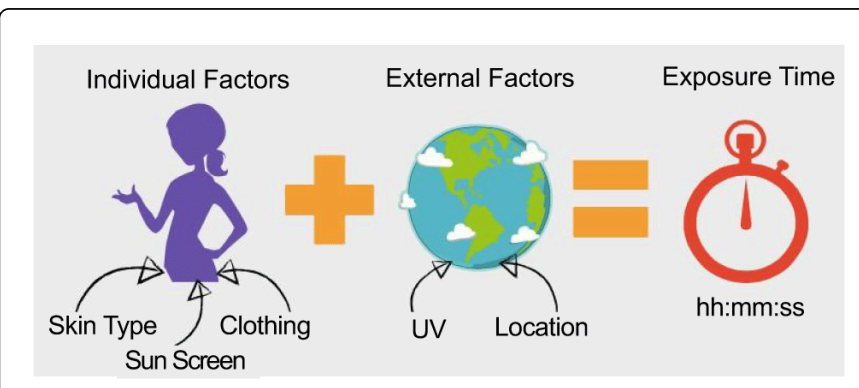

Figure 1: Factors affecting exposure time required for cutaneous vitamin D vitamin D synthesis.

With vitamin D deficiency being associated with bone loss; poor dental health; lower muscle strength; osteomalacia; hyperparathyroidism and perhaps neurocognitive dysfunction and mental illness, particularly depression [12-20], improving vitamin D status is a high priority. Young women are in need of intervention as deficiency in youth affects the "ability to achieve (optimal) potential peak bone mass" [15], increasing the risks of osteoporosis, falls and osteoporotic fractures later in life $[12,15,18]$. Furthermore, women tend to have smaller, thinner bones and are more likely to develop osteoporosis due to sexual differences in skeletal development and greater bone loss with ageing due to menopause [20].

In Australia, skin cancer awareness is prominent, resulting in low vitamin D levels despite abundant UV, as, on the whole, Australians have adopted the Cancer Council's 'slip-slop-slap' campaign [21] very well, inhibiting vitamin D synthesis. Currently, the message to the public is confusing: sunlight is needed for vitamin D yet agencies, including the Cancer Council, urge individuals to limit "exposure to reduce the risk of melanoma" [13]. This is what we refer to as 'complex messaging': there are many variables to consider and conflicting recommendations. Vitamin D deficiency is diagnosed and managed through GPs, however a survey conducted by Bonevski et al. [14] found that advice patients received differed from expert recommendations. Moreover, we found that young women aged
16-25, the target audience, wanted information regarding required sun exposure (77\%); and $68 \%$ believed an app could help improve vitamin $D$ levels and identified a need for better information than their GP currently provided.

All factors affecting vitamin D must be considered by Safe-D to provide appropriate recommendations and education to achieve sufficient exposure. The difficulty here lies in the challenge to balance adequate UV exposure to stimulate sufficient vitamin D synthesis in the skin against the harmful levels that increase risk of melanoma and other skin cancers. As the factors affecting vitamin D synthesis (locational UV, clothing and sunscreen use) are constantly changing and individual factors (skin type) vary, person-to-person messages become additionally complex; messages they must be personalized to the individual at the time of UV-exposure. Safe-D was required to then cater for non-static messages that differ based on the individual factors and the environmental factors at the time of UV-exposure.

\section{Materials and Methods}

This paper reports the two relevant phases of the project: A web survey and participatory action research. In the web survey target population (young women aged 16-25) was surveyed to determine what young women wanted in a Health App. Participatory action research involved three steps, 1) working in a multidisciplinary team to determine a library of appropriate and relevant health messages. 2) Informal focus groups, an advisory private Facebook group and informal 'guerilla testing'. This type of testing involved asking young women approached randomly in public places for their opinions regarding Safe-D message design and 3) Beta testing the delivery and reception of these messages.

First, a small web survey was conducted to understand what the target population, young females, wanted in an app. The survey was advertised via various health channels, a media release and an email to past study participants. Of the respondents $(n=57), 98 \%$ (56) owned a Smartphone, supporting the delivery method of a health app to the target population. Eligible participants were females aged 18-25 residing in Victoria who owned a smartphone. Similar numbers of respondents came from the two age categories: $47 \%$ in the $18-21$ year age group and $53 \%$ in the $22-25$ year age group. However, minor differences existed within the individual ages of the participants with no 18-year-olds responding.

Second, messages in the Safe-D app were developed using participatory action research involving core researchers from both the health domain and human computer interaction domain; a statistician; subject matter expert clinicians; external developers; and target users, to identify key health messages regarding safe levels of UV exposure. Messages were constructed by study members with vitamin D knowledge, using current information from approved Cancer Council Victoria websites where appropriate, deconstructing messages and making them relevant for the target audience by working with clinicians and target users. Experts reviewed all final written copy and Cancer Council sources are referenced where appropriate to reinforce the validity of the information and messages were framed positively to avoid reprimanding users.

Third, during message construction, proposed messages were validated through testing with members of the target population. The design involved healthcare consumers directly, sometimes an underutilized resource in healthcare, to create messages that would resonate with them. 
Page 3 of 7

Last, the app was beta tested using core team members, with Apple and Android devices. Additional beta testers were seconded from the larger YFHI team to ensure a range of platform and operating system configurations for greatest coverage. Beta testing is the final stage of testing software before it is released [21]. The use of beta testing is common practice and acts to validate the app and eliminate any issues before release [22]. In this phase 24 testers, including 6 from the target demographic, were given access to Safe-D to ensure the app behaved in line with user expectations, messages were delivered at opportune times and messages were understood.

\section{Results}

\section{Web survey results}

We found that many young women aged 16-25 years wanted information regarding required sun exposure $(77 \%)$ and identified a need for better information than their GP currently provided. Fortysix percent of respondents reported one notification per day as acceptable from an app. Specifically, 94\% were knowledgeable about the function of vitamin $\mathrm{D}$ in the body. The vast majority identified the link between vitamin D and the sun, and between vitamin D and calcium absorption. Some identified that deficiency is common in Australia and many were aware of their own vitamin D levels. Others identified links with vitamin $\mathrm{D}$ and a wide range of health conditions, signaling that an educated audience was completing the survey. The respondents were most interested in how long they needed to spend in the sun for adequate vitamin D production, the symptoms of low vitamin $\mathrm{D}$ status and foods that were high in vitamin $\mathrm{D}$. The survey enabled identification of factors that the target audience believed would help improve vitamin $\mathrm{D}$ status: reminders; increased understanding of vitamin D and consequences of deficiency; exposure calculations; incorporation of games for motivation. Data regarding the desired information from the 47 respondents who had heard of vitamin $\mathrm{D}$ is in Table 1.

\begin{tabular}{|l|l|l|}
\hline Desired Information & N & $\%$ \\
\hline Science behind vitamin D synthesis & 15 & 32 \\
\hline Reminders about the foods I can eat to get vitamin D & 32 & 68 \\
\hline
\end{tabular}

\begin{tabular}{|c|c|c|}
\hline Scenario & SunSmart Message & Safe-D Equivalent Message \\
\hline $\begin{array}{l}\text { General message about UV exposure } \\
\text { impacts }\end{array}$ & $\begin{array}{l}\text { In all instances, a generic message is } \\
\text { delivered up front; For your vitamin } D \\
\text { today, aim for } 30 \text { minutes (with equivalent } \\
\text { of face, arms and hands exposed) during } \\
\text { the middle of the day. }\end{array}$ & $\begin{array}{l}\text { Messages are delivered based on individual's circumstances. A personalized } \\
\text { calculation that counts down until exposure is met, then counts up to indicate } \\
\text { over exposure. }\end{array}$ \\
\hline $\begin{array}{l}\text { If the day has a high UV alert sun } \\
\text { protection is required push to individual }\end{array}$ & $\mathrm{N} / \mathrm{A}$ & $\begin{array}{l}\text { Today's UV is expected to be high. Look after yourself while outdoors - take } \\
\text { notice of the Safe-D exposure commendations and seek shade once you've } \\
\text { reached your target! }\end{array}$ \\
\hline $\begin{array}{l}\text { If the individual has achieved a safe } \\
\text { level of UV-exposure (determined by } \\
\text { manual entry in SunSmart and a timer in } \\
\text { Safe-D) }\end{array}$ & $\begin{array}{l}\text { Well done! You appear to getting some } \\
\text { sun, which can help with your vitamin D. }\end{array}$ & $\begin{array}{l}\text { Well done, you have received your daily dose of vitamin D. Stop the timer and } \\
\text { slip, slop, slap, seek and slide for the rest of the day. }\end{array}$ \\
\hline $\begin{array}{l}\text { If the day has a low UV no sun } \\
\text { protection is required (low UV alert) - } \\
\text { push to individual }\end{array}$ & $N / A$ & $\begin{array}{l}\text { Today's UV level is expected to be very low. Feel free to spend as much time in } \\
\text { the sun as you like! }\end{array}$ \\
\hline $\begin{array}{l}\text { If the individual has not met UV- } \\
\text { exposure for the day (determined by }\end{array}$ & $\begin{array}{l}\text { You may not be getting enough sun } \\
\text { exposure for Vitamin } D\end{array}$ & $\begin{array}{l}\text { Your sunflower didn't quite get enough sun today. To help your body make } \\
\text { more Vitamin D, try to meet your sun exposure requirements daily. }\end{array}$ \\
\hline
\end{tabular}

\begin{tabular}{|l|l|l|}
\hline $\begin{array}{l}\text { How long I need to be in the sun for adequate vitamin D } \\
\text { production }\end{array}$ & 36 & 77 \\
\hline How to be SunSmart whilst still getting vitamin D & 26 & 55 \\
\hline Symptoms of low vitamin D & 34 & 72 \\
\hline Vitamin D requirements of different population groups & 29 & 62 \\
\hline Where I can get more information about vitamin D & 5 & 11 \\
\hline Nothing - I'm not that interested in vitamin D & 5 & 11 \\
\hline
\end{tabular}

Table 1: Desired Vitamin D Information.

Overall, 32 out of 47 people (68\%) believed an app could help improve their vitamin D levels through (i) UV-exposure (59\%); (ii) providing calculations to communicate the required UV-exposure for vitamin D production (34\%), and (iii) providing information regarding sources of vitamin D (19\%).

\section{Safe-D app results}

The developed app enabled tracking of UV-exposure, recording missed exposure, monitoring progress and simple delivery of complex messages, both automatically and triggered by the study team. Safe-D is discussed below in terms of 1) constructing relevant messages; 2) personalizing messages, and; 3 ) delivering messages.

Constructing Relevant Messages: After analyzing the messages delivered in the SunSmart health app, an app provided by the Cancer Council that includes vitamin D information, it was determined that Safe-D required more complex messaging. SunSmart offers simplistic messaging, informing users of sun protection requirements that are sent to all users of the same skin type. Leveraging inspiration from SunSmart messages Safe-D messages were adapted to be specific and tailored and delivery times were devised for context awareness and active behavior prompting. Safe-D messages are: personalized; pushed to the user rather than requiring them to check the app; written to trigger behavior and improve learning [23-25]. Table 2 illustrates actual scenarios that both Safe-D responds to and compares the difference between simplistic and complex messaging.

Safe-D Equivalent Message

Messages are delivered based on individual's circumstances. A personalized calculation that counts down until exposure is met, then counts up to indicate Today's UV is expected to be high. Look after yourself while outdoors - take notice of the Safe-D exposure commendations and seek shade once you've messages delivered in SunSmart with those in Safe-D to illustrate the 


\begin{tabular}{|l|l|l|}
\hline $\begin{array}{l}\text { manual entry in SunSmart and a timer in } \\
\text { Safe-D) }\end{array}$ & \\
\hline $\begin{array}{l}\text { When the timer is running and indicates } \\
\text { that UV- exposure has exceeded safe } \\
\text { recommendation - push to individual }\end{array}$ & N/A & $\begin{array}{l}\text { Are you still in the sun? Make sure you stop the timer when you have reached } \\
\text { your sun exposure target. The current UV level has exceeded 8. At this time of } \\
\text { the day, it may be unsafe for you to be outside in direct sunlight. Protect } \\
\text { yourself from the sun. Stay indoors and keep well hydrated for now. }\end{array}$ \\
\hline $\begin{array}{l}\text { User did not record UV-exposure } \\
\text { yesterday }\end{array}$ & N/A & $\begin{array}{l}\text { Your sunflower is confused. It isn't sure how much sun you got yesterday. } \\
\text { Please log in and enter your sun exposure details. }\end{array}$ \\
\hline User was over- exposed yesterday & N/A & $\begin{array}{l}\text { You indicated that you had too much sun yesterday. Log in and let us know } \\
\text { what happened. }\end{array}$ \\
\hline
\end{tabular}

Table 2: SunSmart messages and Safe-D equivalents for various scenarios

SunSmart cycles through 10 different messages, combining them in different ways, while Safe-D has multiple messages displayed in different contexts and based on information that is provided back to Safe-D. SunSmart messages are simplistic, broad guidelines while Safe$\mathrm{D}$ provides personalized messages based on the individual exposure, providing tailored recommendations.

Further, in Safe-D, educational messages are delivered weekly to maintain motivation and interest. These messages were designed to be fun, non-clinical and short. It is important to note that health app messages cannot build on previously received messages as participants can start using the app at different times. As indicated in the survey, messages incorporated information regarding vitamin D and sunlight, vitamin $\mathrm{D}$ and calcium absorption and other sources of vitamin D. Messages encourage UV-exposure and positive behaviors and explain the importance of vitamin $\mathrm{D}$, especially in women, risk factors of deficiency, tips to improve vitamin $\mathrm{D}$ and other 'fun facts' to keep users engaged. Examples of these messages include:

16-25 years of age is a wonderful time to develop healthy practices.

With small lifestyle changes like getting a safe amount of sun and tucking into vitamin D rich foods, you can help improve your bones.

Vitamin D deficiency is becoming common due to the popularity of sun avoidance practices and our modern indoor lifestyle.

Your determination to prevent osteoporosis will inspire women everywhere!

Personalizing Messages: All messages in Safe-D are targeted to the specific user of the installation of the health app to improve persuasion, thus outcomes, of receiving the messages. To use Safe-D, the users must answer questions to determine their skin type [26]. Once this is done the user enters clothing coverage and sunscreen use at the time of exposure and Safe-D retrieved real time information from external sources (general positioning data, GPS, and UV levels from the Bureau of Meteorology and the Australian Radiation Protection and Nuclear Safety Agency) to personalize messages. These messages fall into three categories: automatic push notifications; automatic in-app messages; tailored mail messages.

Push notifications are automatically sent to the user when they need to perform an action. These notifications garner attention to activate the user to perform the behavior as per the Fogg Behavioural Model, triggering said behavior to occur [26].

Messages also automatically display within the app when action is recorded or feedback is required to aid in decision-making and education. For example, these messages communicate to the user whether they have reached or exceeded their tailored UV-exposure recommendation. Tailored mail messages can be sent to defined groups. If the user recorded that they could not use Safe-D, and provided the reason, this can be used to construct sensitively tailored messages; for example, if an individual is sick they will not be told to get exposure, thereby reducing guilt. However, if they select 'too busy' or 'indoors all day' it is possible for the study team to send tailored communications via mail to encourage short exposure. Messages can be sent to groups to improve effectiveness rather than generic messages sent to all; other messages are interactive, based on user inputs.

Delivering Complex Messages: Turning the complex and confounding sun exposure factors into an algorithm is essential to deliver safe, personalized recommendations. The mechanics of this algorithm, however, are not apparent to users; it is the messages themselves that convey the complex information simply, and the use of gamification to further simplify understanding. Gamification is the use of game elements in non-game contexts to provoke competition [27] for behavior change and to convey messages simply and effectively. Gamification in Safe-D relates UV-exposure back to a sunflower, whose health the users are responsible for through safe UVexposure recorded. The complex messaging occurs on a per scenario basis, as different messages are handled differently. Messages can be delivered in one, or multiple modalities: in-app; mail; push notifications. In-app messages deliver point-of-interaction information through avoidance of messaging at inopportune times and provision of immediate feedback. Mail messages include tailored communications, extreme UV warnings and weekly educational notifications. Push notifications are automatically delivered when behavior is required.

The Safe-D team acknowledges that safety is paramount and advises Safe-D app users always to apply their own commonsense and judgment if the app seems to provide inappropriate sun exposure advice. This circumstance might arise for example if there is a rapid and unexpected weather change that abruptly increased the UV index and potential hazard associated with outdoor activity.

\section{Focus Group Results}

Testing messages with target users in the focus groups and an informal Facebook group revealed that young women wanted information to explain the medical consequences and risks of too much sun exposure. While they wanted this information, they wanted the messages to be cautioning and less passive while being fun and interesting rather than scary. Users identified that it would be useful to have different messages every day if the behavior required from them is going to be the same, since they would get bored seeing the same 
message several days in a row. Given this feedback, minor changes were made to messages to make them more interesting and nonrepetitive; messages were cycled through different wording to avoid monotony.

\section{Beta Testing Results}

The beta testing phase enabled deeper understanding of the timing of triggering messages. While the preliminary survey indicated that one notification per day was acceptable (46\%), beta testing revealed that one per day, when the user wasn't actively using Safe-D, was annoying and could potentially inhibit use. Push notifications only send when: behaviour needs to occur; to prompt app use; an adverse health event was recorded yesterday, and a weekly educational message. The timing of exposure-related notifications was designed to be during use, to not occur at inopportune times. Similarly, warning notifications only occur twice as constant prompts may lead to annoyance.

\section{Discussion}

It is important for health service providers to explore the potential for personalized health message delivery. We recommend that messaging should be:

Tailored per user, based on user input, external real-time data and interactions with the app;

Calibrated and cleaned with target users to ensure understanding of the intended message;

Made available for later retrieval to reinforce education;

Pushed from a central server so that messages may be customized and changed without requiring users to update the app;

Able to be customized and delivered to user sub-sets from the central server, and;

Tied to gamification to reinforce learning by leveraging a heuristic to prompt for behavior when not using the app.

Further, the modality in which the messaging should occur depends on the scenario. Instant feedback is required while the user is interacting with the health app, thus should be provided within the app itself. When behavior needs to occur, messages should be pushed to the user, using push notifications and available within the app. When the user has not been interacting with the app for a defined time, a message should be pushed to the user to encourage them to use the app again. When general education messages are provided, or personalized and individualized information is sent outside of normal app interaction, these should be pushed to the user and available for later retrieval. When these principles are applied to developing a health app, messages can be personalized to an individual and sent at relevant times in an appropriate modality. This is highlighted in the differences between SunSmart and Safe-D as outlined in Table 3. It must be noted that SunSmart provides many additional functions than Safe- D regarding sun behavior; SunSmart's Vitamin D Tracker is a small component of the overall app and was not designed for targeted health service delivery as Safe-D was. This comparison illuminates the differences in health apps designed for simplistic messaging and those designed for complex targeted messaging.

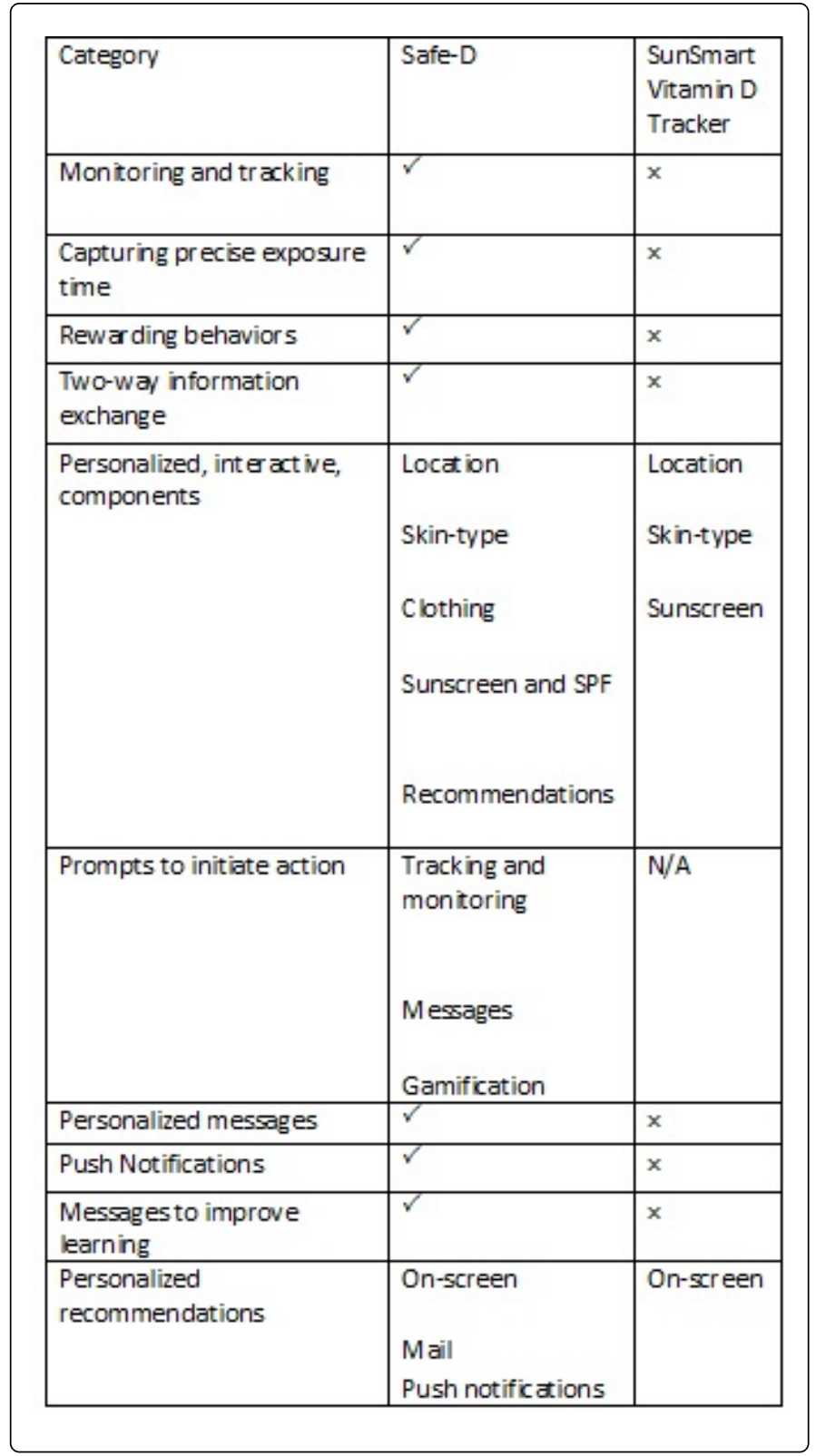

Table 3: Safe-D and SunSmart comparison.

Messaging in SunSmart is dependent on the user actively 'pulling' them from the app, messages are displayed by interacting with the app, while Safe-D employs a mixture of pull and push messages based on the context. For example, when the user is actively exposing them to $\mathrm{UV}$, Safe-D is able to provide her with real time updates that she has met or exceeded targets, and push safety warnings when UV is extreme. In SunSmart, all recommendations are displayed within the app when the user goes through the process of entering estimated time in the sun and estimated skin exposed. The skin exposure options in SunSmart are basic as the most a user can expose is face, arms and hands, providing one-way messaging. When users have not being using SunSmart, no action occurs from the health app; however, in Safe-D reminders are sent to prompt behavior change. Safe-D is interactive and simplifies vitamin D complexities to two simple entry 
points, providing recommendations and gamification to simplify the message of safe UV-exposure.

Safe-D then provides information back in the form of gamification and tailored and personalized messages, reinforced by a sunflower metaphor. SunSmart uses information regarding UV at the time the user estimates that they will go in to the sun and provides simplistic messages based on skin type upon interaction with the app.

\section{Generalizability}

Potentially most conditions will require individualized and complex messaging with the move towards personalized medicine [28-31]. As these principles do not encompass a set procedure, but rather recommended guidelines, generalizability is not considered an issue.

\section{Limitations and Future Directions}

This research formulated principles to guide development of health apps to support targeted complex health messages. It has not, for example, included principles to tailor the messages themselves based on context-specific factors such as message differences required for developed versus developing areas or different cultural and socioeconomical groups.

Findings are based on a reflective analysis from Participatory Action Research, it is possible that the guidelines may have inherent bias. This was mitigated somewhat through the triangulation of data, including beta testers who were not involved directly involved in the research. As the Safe-D app is currently under evaluation in a randomized controlled trial (ACTRN12613000972729) the results are at this stage largely theoretical and require validation before their suitability can be entirely understood. The lack of evaluation is a limitation to these principles; while behavior change was experienced anecdotally among the core researchers and beta testers, the greater Safe-D study will act to validate the app and therefore principles. Furthermore, future work in the e-health app arena should leverage these principles to validate them.

\section{Conclusion}

This research has provided the tangible output of Safe-D, currently under evaluation in a randomized controlled trial (RCT; ACTRN 12613000972729), and on a theoretical level, insights into how to deliver complex messages to young women via the app. Working with clinicians, researchers from both the health domain and human computer interaction and target users collaborated to create messages, enabled lessons learned to be leveraged to create a set of guidelines for message delivery, presented in this paper. The RCT will further evaluate whether these messages are more effective than traditional, brochure- based information.

\section{Acknowledgement}

This paper is on behalf of the Safe-D Study group: Professor John Wark, Dr. Shanton Chang, Dr. Nicola Reavley, Associate Professor Marie Pirotta, Professor George Varigos, Professor Kim Bennell, Professor Suzanne Garland, Ms. Alexandra Gorelik, Professor Anthony Jorm, Dr. Tharshan Vaithianathan, Ms. Emma Callegari, Ms. Stefanie Hartley, Ms. Skye Maclean, Ms. Nazanin Shajiei, Ms. Audrey Grech, Ms. Anna Scobie, Miss. Kayla Heffernan, Ms. Amber Bourgeois Ms. Karen Gillett and Ms. Ashleigh Buckland. Safe-D was developed by Boosted Human: Mr. Ashemah Harrison and Mr. Thomas Ruffie.
Safe-D is funded by a National Health and Medical Research Council (NHMRC) grant APP1049065.

\section{References}

1. Free C, Phillips G, Galli L, Watson L, Felix L, et al. (2013) The effectiveness of mobile-health technology-based health behaviour change or disease management interventions for health care consumers: a systematic review. PLoS Med 10: e1001362.

2. Free C, Phillips G, Felix L, Galli L, Patel V, et al. (2010) The effectiveness of M-health technologies for improving health and health services: a systematic review protocol. BMC Res Notes 3: 250.

3. Terry M (2010) Medical Apps for Smartphones. Telemed J E Health 16: 17-22.

4. Research2Guidance (2013) Mobile Health Market Report 2013-2017. The Commerzialization of mHealth Apps.

5. McCartney M (2013) How do we know whether medical apps work? BMJ 346: f1811.

6. Levine BA, Goldschlag D (2013) Apps and Monitors for Patient Health: Apps for Fitness, Diet, and Sleep Help Patients Quantify their Activity and Reach their Goals.

7. Breton ER, Fuemmeler BF, Abroms LC (2011) Weight loss-there is an app for that! But does it adhere to evidence-informed practices? Transl Behav Med 1: 523-529.

8. Ijsselsteijn W (2006) Persuasive Technology: First International Conference on Persuasive Technology for Human Well-Being. Communications of the Association for Information Systems, 24.

9. Borsboom AS (2014) Investigating the potential of Interactive Media to Encourage Engagement with Type 1 Diabetes Management. Conferences in Research and Practice in Information Technology (CRPIT), 153.

10. Consolvo S, Landay JA (2009) Designing for Behavior Change in Everyday Life. IEEE Computer Society, 405, 86-89.

11. Dennison L, Morrison L, Conway G, Yardley L (2013) Opportunities and challenges for smartphone applications in supporting health behavior change: qualitative study. J Med Internet Res 15: e86.

12. Holick MF (2008) Vitamin D: a D-Lightful health perspective. Nutr Rev 66: S182-194.

13. Green T, Li W, Whiting S (2013) Strategies for Improving Vitamin D Status: Focus on Fortification. Nutritional Influences on Bone Health. Springer London, 247-260.

14. Mithal A, Wahl DA, Bonjour JP, Burckhardt P, Dawson-Hughes B, et al. (2009) Global vitamin D status and determinants of hypovitaminosis D. Osteoporos Int 20: 1807-1820.

15. Barnes MS, Robson PJ, Bonham MP, Strain JJ, Wallace JM (2006) Effect of vitamin D supplementation on vitamin D status and bone turnover markers in young adults. Eur J Clin Nutr 60: 727-733.

16. Cashman K (2010) Effects of Vitamin D on Bone Health in Healthy Young Adults. In: Burckhardt P, Dawson-Hughes B and Weaver C. Nutritional Influences on Bone Health. Springer London.

17. Daly R (2013) Nutrition, Aging, and Chronic Low-Grade Systemic Inflammation in Relation to Osteoporosis and Sarcopenia. In: Burckhardt P, Dawson-Hughes B and Weaver CM. Nutritional Influences on Bone Health. Springer London.

18. Lips P, Schoor N (2013) Physical Performance, Muscle Strength, Falls, and Vitamin D. In: Burckhardt P, Dawson-Hughes B \& Weaver CM. Nutritional Influences on Bone Health. Springer London.

19. Pittas AG, Harris SS, Stark PC, Dawson-Hughes B (2007) The effects of calcium and vitamin D supplementation on blood glucose and markers of inflammation in nondiabetic adults. Diabetes Care 30: 980-986.

20. Orwoll ES, Bliziotes M (1994) Heterogeneity in osteoporosis. Men versus women. Rheum Dis Clin North Am 20: 671-689.

21. Cancer Council (1981). Slip! Slop! Slap! Campaign.

22. Bonevski B, Girgis A, Magin P, Horton G, Brozek I, et al. (2012) Prescribing Sunshine: A Cross-Sectional Survey Of 500 Australian 
Citation: Heffernan KJ, Chang S, Maclean ST, Callegari ET, Garland SM, et al. (2014) The potential of eHealth Apps to Support Targeted Complex Health Messages. J Gen Practice 2: 182. doi:10.4172/2329-9126.1000182

Page 7 of 7

General Practitioners' Practices and Attitudes About Vitamin D International Journal of Cancer 130: 2138-2145.

23. Fitzpatrick TB (1988) The validity and practicality of sun-reactive skin types I through VI. Arch Dermatol 124: 869-871.

24. Fogg B (2009) A Behavior Model for Persuasive Design. ACM Proceedings of the 4 th International Conference on Persuasive Technology 40 .

25. Fine MR (2002) Beta testing for better software. John Wiley and Sons.

26. Dolan RJ, Matthews JM (1993) Maximizing the utility of customer product testing: beta test design and management. Journal of Product Innovation Management 10: 318-330.

27. Kapp KM (2012) The Gamification of Learning and Instruction: Gamebased Methods and Strategies for Training and Education, John Wiley and Sons.
28. Husereau D, Marshall DA, Levy AR, Peacock S, Hoch JS (2014) Health Technology Assessment and Personalized Medicine: Are Economic Evaluation Guidelines Sufficient to Support Decision Making? International Journal of Technology Assessment in Health Care 1-9.

29. Katsios C, Roukos DH (2010) Individual Genomes and Personalized Medicine: Life Diversity and Complexity. Personalized Medicine, 7: 347-350.

30. Meric-Bernstam F, Farhangfar C, Mendelsohn J, Mills GB (2013) Building a Personalized Medicine Infrastructure at A Major Cancer Center. Journal of Clinical Oncology, 31: 1849-1857.

31. Kwasky AN, Groh CJ (2012) Vitamin D and depression: is there a relationship in young women? J Am Psychiatr Nurses Assoc 18: 236-243. 